\title{
Fatores de risco para infecção de sítio cirúrgico em cirurgias traumato- ortopédicas
}

Risk factors and surgical site infection in trauma-orthopedic surgeries

Factores de riesgo e infección del sitio quirúrgico en cirugías ortopédicas y traumatológicas

Como citar este artigo

Silva, Edilane Neves da; Silva, Renata Kelly dos Santos e; Carvalho, Simone Barroso de; Façanha, Dilene Maria de Araújo; Carvalho, Rhanna Emanuela Fontelene Lima de; Pereira, Francisco Gilberto Fernandes. Fatores de risco para infecção de sítio cirúrgico em cirurgias traumato-ortopédicas. Revista Cuidarte. 2021;12(2):e1292.

http://dx.doi.org/10.15649/cuidarte.1292

Revista Cuidarte

Rev Cuid. May - Ago 2021; 12(2): e1292

doi) http://dx.doi.org/10.15649/cuidarte.1292

E-ISSN: 2346-3414

(1) Edilane Neves da Silva ${ }^{1}$

(1) Renata Kelly dos Santos e Silva²

(1) Simone Barroso de Carvalho ${ }^{3}$

(1) Dilene Maria de Araújo Façanha ${ }^{4}$

(1) Rhanna Emanuela Fontelene Lima de Carvalho ${ }^{5}$

(1) Francisco Gilberto Fernandes Pereira $^{6}$

1 Universidade Federal do Piaui, Brasil. E-mail:

lane_silva_rso@hotmail.com

2 Universidade Federal do Piaui, Brasil.E-mail: r.ks@hotmail.com

3 Hospital Regional Justino Luz, Brasil. E-mail: simonebcarvalho2009@ hotmail.com

4 Universidade Federal do Ceará, Brasil. E-mail:

dilenefacanha@gmail.com

5 Universidade Estadual do Ceará, Brasil. E-mail: rhannalima@uece.br

6 Universidade Federal do Piaui, Brasil. Autor correspondente E-mail: gilberto.fp@hotmail.com

\section{Resumo}

Introdução: A Infecção do Sítio Cirúrgico ocupa a terceira posição entre as infecções adquiridas em serviços de saúde, configurando-se como um importante problema de saúde pública, presente em cerca de $15 \%$ daquelas encontradas em pacientes hospitalizados, e um dos tipos de cirurgias de maior probabilidade para esse evento são as traumato-ortopédicas. Objetivo: Verificar a associação entre os fatores de risco e a presença de Infecção de Sítio Cirúrgico em cirurgias traumato-ortopédicas. Materiais e métodos: Estudo prospectivo descritivo, quantitativo, realizado entre agosto a outubro de 2017 com 84 pacientes. Os dados referentes ao paciente, ao procedimento e os sinais de Infecções de Sítio Cirúrgico, encontrados no terceiro e décimo dia de pós-operatório, foram coletados por meio de um formulário. Resultados: Das variáveis estudadas, comportaram-se como fatores de risco de Infecção de Sítio Cirúrgico: tabagismo, diabetes e idade acima de 50 anos. A idade foi o principal fator de risco relacionado ao paciente, presente em 31, 36,9\% dos casos. Dor, edema e hiperemia foram os sinais de infecção mais prevalentes. Discussão e conclusões: Os fatores de risco relacionados ao paciente possuem maior relevância na associação com infecção do sítio cirúrgico em comparação com os relacionados ao procedimento.

Palavras chave: Fatores de Risco; Infecção Hospitalar; Ferida Cirúrgica; Ortopedia; Enfermagem Perioperatória.
Recebido: junhio 08 de 2020

Aceito: 22 fevreiro de 2021

Publicado: abril 30 de 2021 $\square *$ Correspondencia

Francisco Gilberto Fernandes Pereira

E-mail: gilberto.fp@hotmail.com 


\section{Risk factors and surgical site infection in trauma-orthopedic surgeries}

\section{Abstract}

Introduction: Infection of the Surgical Site occupies the third position among infections acquired in health services, representing an important public health problem, present in about $15 \%$ of those found in hospitalized patients, and one of the types of most likely for this event are trauma-orthopedic surgies. Objective: To verify the association between risk factors and the presence of Surgical Site Infection in traumato-orthopedic surgeries. Materials and methods: Prospective descriptive, quantitative study, conducted between August and October 2017 with 84 patients. The data regarding the patient, the procedure and the signs of Surgical Site Infections, found on the third and tenth postoperative days, were collected using a form. Results: Of the studied variables, they behaved as risk factors for Surgical Site Infection: smoking, diabetes and age over 50 years. Age was the main risk factor related to the patient, present in 31, 36.9\% of cases. Pain, edema and hyperemia were the most prevalent signs of infection. Discussion and conclusions: The risk factors related to the patient are more relevant in the association with infection of the surgical site compared to those related to the procedure.

Keywords: Risk Factors; Cross Infection; Surgical Wound; Orthopedics; Perioperative Nursing.

\section{Factores de riesgo e infección del sitio quirúrgico en cirugías ortopédicas y traumatológicas}

\section{Resumen}

Introducción: La infección del sitio quirúrgico ocupa el tercer lugar entre las infecciones adquiridas en los servicios de salud, lo que representa un problema importante de salud pública, presente en aproximadamente el $15 \%$ de las que se encuentran en pacientes hospitalizados, y uno de los tipos de lo más probable para este evento son las cirugías traumatología ortopédica. Objetivo: Verificar la asociación entre los factores de riesgo y la presencia de infección del sitio quirúrgico en cirugías traumatológicas. Materiales y métodos: Estudio prospectivo descriptivo, cuantitativo, realizado entre agosto y octubre de 2017 con 84 pacientes. Los datos sobre el paciente, el procedimiento y los signos de infecciones del sitio quirúrgico, encontrados en el tercer y décimo día postoperatorio, se obtuvieron mediante un formulario. Resultados: De las variables estudiadas, se comportaron como factores de riesgo para la infección del sitio quirúrgico: tabaquismo, diabetes y edad mayor de 50 años. La edad fue el principal factor de riesgo relacionado con el paciente, presente en $31,36,9 \%$ de los casos. El dolor, el edema y la hiperemia fueron los signos más frecuentes de infección. Discusión y conclusiones: Los factores de riesgo relacionados con el paciente son más relevantes en la asociación con la infección del sitio quirúrgico en comparación con los relacionados con el procedimiento.

Palabras clave: Factores de Riesgo; Infección Hospitalaria; Herida Quirúrgica; Ortopedia; Enfermería Perioperatoria. 


\section{Introdução}

As Infecções Relacionadas à Assistência à Saúde (IRAS) são decorrentes de procedimentos que podem ser colonizados por micro-organismos, e culminam no aumento na morbimortalidade bem como no tempo de internação dos pacientes ${ }^{1}$. No Brasil, a Infecção do Sítio Cirúrgico (ISC) ocupa a terceira posição entre as infecções em serviços de saúde, compreendendo cerca de $15 \%$ daquelas encontradas em pacientes hospitalizados ${ }^{2}$.

A ocorrência dessas infecções representa um importante problema de saúde pública, configurando-se como causa primordial na ocorrência de iatrogenia aos pacientes submetidos a intervenções invasivas ${ }^{3}$, como é o caso das cirurgias traumato-ortopédicas. Em vista disso, sua alta incidência e efeitos deletérios demandam monitorização sistemática desses pacientes, visando o reconhecimento precoce de fatores de risco para ISC e a implementação

A ocorrência dessas infecções representa um importante problema de saúde pública, configurando-se como causa primordial na ocorrência de iatrogenia aos pacientes submetidos a intervenções invasivas ${ }^{3}$, como é o caso das cirurgias traumato-ortopédicas. efetiva de medidas de suporte às práticas de segurança do paciente.

O diagnóstico de ISC baseia-se nos sinais de infecção em 30 a 90 dias após o procedimento cirúrgico ou até um ano depois nos casos de implantes de órteses e próteses ${ }^{2,4}$. Nos casos em que há necessidade de implantes ou utilização temporária de próteses, a ocorrência de ISC torna-se especialmente comum. Portanto, as cirurgias traumato-ortopédicas se enquadram neste perfil, pois possuem como agravante o fato de que geralmente são indicadas nos casos de acidentes em lesão que já se apresenta contaminada ou potencialmente contaminada na chegada ao serviço hospitalar, bem como, dada a urgência, muitas vezes não há uma avaliação prévia dos fatores de risco ${ }^{1,5}$.

As ISC ocasionam diversos transtornos de ordem econômica e social, com a diminuição da rotatividade de leitos, risco aumentado em três vezes de readmissão hospitalar e óbito ${ }^{6-7}$. Somado ao fato de que a etiologia da infecção está amplamente relacionada a fatores ambientais, individuais e técnicos, um enorme desafio é lançado para as instituições hospitalares quanto à eficiência de suas medidas de controle e prevenção ${ }^{8}$.

Sendo um procedimento invasivo, a cirurgia ortopédica desponta como facilitadora no desencadeamento de ISC, o que demanda rigor em todo o processo perioperatório. Nesse ínterim, os fatores de risco associados à infecção

Os fatores de risco associados à infecção ortopédica podem estar relacionados ao próprio paciente, à execução da técnica cirúrgica e ao ambiente onde será realizado o procedimento cirúrgico. ortopédica podem estar relacionados ao próprio paciente, à execução da técnica cirúrgica e ao ambiente onde será realizado o procedimento cirúrgico.

Dessa forma, a literatura aponta que os principais fatores de risco para ISC em cirurgias ortopédicas são: a condição clínica do paciente ${ }^{5,7}$; diabetes mellitus, obesidade, idade ${ }^{6,8-9}$, internação pré-operatória prolongada ${ }^{5,9}$, antissepsia da pele; técnica de degermação e uso de Equipamentos de Proteção Individual (EPI's) pela equipe ${ }^{7,10}$ e duração da cirurgia ${ }^{8-10}$.

Dada sua significância para os serviços de saúde, em 2009 a Organização Mundial da Saúde (OMS) através da Aliança Mundial para a Segurança do Paciente, lançou como um dos objetivos essenciais para a cirurgia segura o fato de que a equipe deve usar sistematicamente métodos conhecidos para minimizar o risco de infecção do sítio cirúrgico ${ }^{11}$. 
Diante do exposto, questiona-se quais os principaisfatores associados à Infecção do Sítio Cirúrgico em cirurgias traumato-ortopédicas? Portanto, identificar os principais fatores que causam as ISC, justifica-se tanto pelo ponto de vista da saúde do paciente, como no aspecto financeiro, pois são responsáveis pelo aumento da permanência no hospital e consequentemente aumento de custos com o tratamento antimicrobiano, exames e curativos.

Objetivou-se, portanto, verificar a associação entre os fatores de risco e a presença de infecções de Sítio Cirúrgico em pacientes submetidos às cirurgias traumato-ortopédicas.

\section{Materiais e Métodos}

Estudo prospectivo descritivo, com abordagem quantitativa, norteado pela ferramenta STROBE, realizado entre os meses de janeiro a novembro de 2017 em um hospital de médio porte na cidade de Picos, Piauí. A instituição presta assistência generalista englobando pacientes obstétricas, pediátricos, clínica geral e cirúrgica, sendo que para esta última área destinam-se 5 enfermarias e 34 leitos do serviço.

O cálculo amostral foi definido a partir da média mensal de cirurgias traumato-ortopédicas realizadas no ano de 2016 que foi de 106 procedimentos. Adotando o critério de amostragem probabilística com nível de confiança 95\% e nível de precisão de 5\%, participaram do estudo 84 indivíduos que atendiam aos seguintes critérios de inclusão: encontravam-se em período préoperatório de cirurgia ortopédica, havendo possibilidade para contato telefônico após a alta e maiores de 18 anos. Foram excluídos os pacientes que já estavam em pós-operatório ou que se submeteram a cirurgias de emergência.

Ressalta-se que houve perda amostral durante o seguimento do estudo, tendo em vista que 11 pacientes se omitiram a participar de alguma etapa da coleta de dados, mesmo tendo aceitado inicialmente em participar.

Os dados foram coletados entre os meses de agosto a outubro de 2017 por meio da aplicação de um formulário contendo as seguintes informações: dados de identificação, fatores de risco para ISC relacionados ao paciente (idade > 50 anos; estadia pré-operatória prolongada; obesidade; desnutrição; diabetes mellitus; comorbidades; tabagismo; medicamentos de uso contínuo; alergia medicamentosa), fatores de risco para ISC relacionados ao procedimento cirúrgico (duração da cirurgia; preparo da pele do paciente; grau de contaminação da cirurgia; antibioticoterapia profilática; uso de materiais ortopédicos), e detecção dos sinais de ISC (dor ou aumento da sensibilidade local; edema local; hiperemia; drenagem purulenta; deiscência espontânea; abcesso; febre $>38^{\circ} \mathrm{C}$ ) através de perguntas dicotômicas. O instrumento foi formulado baseado nos componentes de vigilância de infecções hospitalares de acordo com a Metodologia National Nosocomial Infection Surveillance System (NNIS).

Foram realizadas quatro visitas semanais durante os três meses na unidade cirúrgica do hospital, de modo que se pudessem contemplar todas as etapas da pesquisa conforme descritas a seguir:

- PRIMEIRA ETAPA: os pacientes foram abordados em suas respectivas enfermarias, sendo convidados a participar da pesquisa e responder ao instrumento de coleta de dados, bem como foram informados sobre os seguimentos posteriores do estudo. Esta etapa se propôs a identificar os fatores de risco para ISC relativos ao paciente, preenchimento dos dados pessoais e teve um tempo estimado de quinze minutos para sua conclusão, de modo que não interferisse nos procedimentos terapêuticos do paciente; 
- SEGUNDA ETAPA: foi realizada no terceiro dia de pós-operatório e se propôs a identificar os fatores de risco para ISC relacionadas ao procedimento cirúrgico e a presença de sinais de ISC. Esta etapa ocorreu ainda na enfermaria em que o paciente estava instalado e obedeceu ao mesmo rigor da etapa anterior. No entanto, para a identificação dos fatores de riscos relacionados ao procedimento, foi realizada uma consulta ao prontuário e à ficha de sala, onde estão registrados os procedimentos adotados durante a cirurgia;

- TERCEIRA ETAPA: foi realizada no décimo dia de pós-operatório. Considerando que alguns pacientes já haviam recebido alta hospitalar, essa etapa também pôde ser realizada por meio de contato telefônico e se propôs a detectar a presença de sinais de ISC. Para não haver distorções com relação a interpretação dos possíveis sinais de ISC, o paciente foi esclarecido, no momento da abordagem inicial, sobre os tipos de sinais mais comuns e como detectá-los, portanto, ele foi capacitado de reportar sua ocorrência na ocasião do contato telefônico. Nos casos de contato telefônico, quando os pesquisadores não obtiveram sucesso em duas tentativas, o paciente foi excluído da pesquisa.

A escolha pelo terceiro e décimo DPO se baseia na constatação de que este intervalo é mais frequente quanto ao surgimento de ISC em cirurgias como as traumato-ortopédicas ${ }^{3}$.

Após a coleta, os dados foram categorizados no programa IBM-Statistics Statistics Package Social Sciences (SPSS) versão 20.0 em que foram realizadas as operações estatísticas descritivas das frequências relativa e absoluta, cálculo das medidas de tendência central e dispersão, Razão de Verossimilhança e o teste do qui-quadrado para verificar a associação entre os fatores de risco e a ISC. Associações estatísticas foram consideradas significativas quando $p<0,05$.

Esta pesquisa seguiu as recomendações da Resolução n. 466/12 do Conselho Nacional de Saúde mediante a aprovação pelo Comitê de Ética em Pesquisa com Seres Humanos da Universidade Federal Piauí.

\section{Resultados}

A caracterização sociodemográfica revelou que dos 84 participantes do estudo, a maioria pertenceu ao sexo masculino $(n=62 ; 73,8 \%)$ com idade variando entre 18 e 92 , e mediana de 39 anos ( $n=68 ; 80,9 \%)$. Estiveram presentes mais participantes com estado civil solteiro ( $n=42$; $50 \%)$, do que casado ou em união estável $(n=37 ; 44 \%)$. Os principais diagnósticos médicos foram de fratura interna $(n=59 ; 70,2 \%)$ e fratura exposta $(n=16 ; 19 \%)$, enquanto o sítio cirúrgico mais citado localizava-se nos membros superiores $(n=46 ; 54,8 \%)$.

Verificou-se que 52 (61,9\%) dos pacientes apresentou Infecção de Sítio Cirúrgico, destacandose que nesta pesquisa os fatores de risco para a ocorrência deste evento foram divididos em: relacionados ao paciente, e relacionados ao procedimento cirúrgico.

Quanto aos fatores de risco para ISC relacionados ao paciente, identificou-se mais de um fator no mesmo indivíduo, sendo o fator mais evidente a idade superior a 50 anos ( $n=31 ; 36,9 \%)$. Em sequência, nota-se a estada pré-operatória superior a quatro dias $(n=27 ; 32,1 \%)$, a relação entre os medicamentos de uso habitual ( $n=24 ; 28,6 \%)$, o tabagismo $(n=21 ; 25 \%)$ e o Diabetes Mellitus $(\mathrm{n}=9 ; 10,7 \%)$, (Tabela 1). 
Tabela 1. Fatores de risco para Infecção de Sítio Cirúrgico relacionados ao paciente. Picos, Piauí, Brasil, 2017.

\begin{tabular}{lcc}
\hline Variáveis & $\boldsymbol{f}^{*}$ & $\%$ \\
\hline Idade $>50$ anos & 31 & $36,9 \%$ \\
Estadia pré -operatória prolongada & 27 & $32,1 \%$ \\
Obesidade & 4 & $4,8 \%$ \\
Desnutrição & 1 & $1,2 \%$ \\
Diabetes mellitus & 9 & $10,7 \%$ \\
Comorbidades & 7 & $8,3 \%$ \\
Tabagista & 21 & $25,0 \%$ \\
Medicamento de uso habitual** & 24 & $28,6 \%$ \\
Alergia medicamentosa & 5 & $6,0 \%$ \\
\hline
\end{tabular}

Nota: $f^{*}$ frequência de casos; \% percentual representado. ** corticoides; anticoagulantes; imunossupressores.

No que se refere aos fatores de risco para ISC relacionados ao procedimento cirúrgico, todos os pacientes apresentaram o preparo da pele e o uso de antibioticoterapia profilática $(n=84 ; 100 \%)$. A maior parte dos antibióticos eram administrados entre 15 a 25 minutos antes da cirurgia, tempo médio três vezes menor do que o atual preconizado.

Outros aspectos significativos foram cirurgia com duração inferior a 2 horas $(n=83 ; 98,8 \%)$ enquanto cirurgias limpas e o uso de materiais ortopédicos incluindo pinos, parafusos e fixadores foram citados 63 vezes (75\%) dentre os 84 procedimentos (Tabela 2). Tanto a duração do procedimento e o implante de materiais proporcionam maiores chances de desenvolver infecção pelo tempo maior de exposição necessário bem como a ruptura de maior superfície corporal, refletindo na extensão da ferida operatória e tempo de cicatrização.

Tabela 2. Fatores de risco para Infecção de Sítio Cirúrgico relacionados ao procedimento cirúrgico. Picos, Piauí, Brasil, 2017.

\begin{tabular}{lcc}
\hline Variáveis & $\boldsymbol{f}^{*}$ & $\%$ \\
\hline Duração da cirurgia & & \\
$\quad$ < horas & 83 & $98,8 \%$ \\
$\quad$ 2 horas & 1 & $1,2 \%$ \\
Preparo da pele do paciente & 84 & $100 \%$ \\
Grau de contaminação da cirurgia & & \\
$\quad$ Limpa & 63 & $75,0 \%$ \\
$\quad$ Potencialmente contaminada & 15 & $17,9 \%$ \\
Contaminada & 5 & $6,0 \%$ \\
$\quad$ Infectada & 1 & $1,2 \%$ \\
Antibioticoterapia profilática & 84 & $100 \%$ \\
Uso de materiais ortopédicos & 63 & $75,0 \%$ \\
\hline
\end{tabular}

Nota: $f^{*}$ frequência de casos; \% percentual representado. Mais de um fator de risco pode ter sido encontrado no mesmo paciente. 
Acerca das variáveis relacionadas aos sinais e sintomas de ISC, evidenciaram que o edema e a dor foram os mais comuns no $3^{\circ} \mathrm{DPO}$ e $10^{\circ} \mathrm{DPO}$. Hiperemia foi o terceiro sinal mais citado em ambas ocasiões com frequência de 24 (28,6\%) no $3^{\circ}$ DPO, e $18(21,4 \%)$ no $10^{\circ}$ DPO, o que demonstra haver compatibilidade entre os sintomas de ISC apresentados ao longo do pósoperatório, exceto pela presença de deiscência espontânea $(n=2 ; 2,4 \%)$ no $10^{\circ}$ DPO, pois trata-se de um indicativo de complicações severas relacionada a ISC (Tabela 3).

De modo semelhante, a ocorrência de drenagem purulenta se fez mais presente durante o $10^{\circ}$ DPO, também como indício de infecções em estágio mais avançado, diferente do aumento de sensibilidade sentido mais evidentemente no $3^{\circ}$ DPO como sintoma imediato que pode ser provocado pelo procedimento cirúrgico ou mesmo pelo trauma sofrido antes da cirurgia.

A febre ocorre como uma forma sistêmica do organismo auxiliar no combate a agentes invasores do sistema imune, podendo se manifestar em qualquer época do processo infeccioso. Nesta amostra, a febre foi percebida em maior grau no $3^{\circ}$ DPO $(n=10 ; 11,9 \%)$ em relação ao $10^{\circ}$ DPO $(\mathrm{n}=7 ; 8,3)$, como pode ser observado na Tabela 3.

Tabela 3. Sinais e sintomas de Infecção de Sítio Cirúrgico apresentados pelos pacientes no $3^{\circ} \mathrm{e}$ 10 Dia de Pós-Operatório. Picos, Piauí, Brasil, 2017.

\begin{tabular}{lcccc}
\hline \multirow{2}{*}{ Variáveis } & \multicolumn{2}{c}{$\mathbf{3}^{\circ} \mathbf{D P O}$} & \multicolumn{2}{c}{$\mathbf{1 0}^{\circ} \mathbf{D P O}$} \\
\cline { 2 - 5 } & $\boldsymbol{f}^{*}$ & $\%$ & $\boldsymbol{f}^{*}$ & $\%$ \\
\hline Dor ou aumento da sensibilidade & 44 & $52,4 \%$ & 38 & $45,2 \%$ \\
Edema local & 40 & $47,6 \%$ & 21 & $25,0 \%$ \\
Hiperemia & 24 & $28,6 \%$ & 18 & $21,4 \%$ \\
Drenagem purulenta & 5 & $6,0 \%$ & 6 & $7,1 \%$ \\
Deiscência espontânea & & & 2 & $2,4 \%$ \\
Abscesso & 1 & $1,2 \%$ & 1 & $1,2 \%$ \\
Febre $\left(>38^{\circ}\right)$ & 10 & $11,9 \%$ & 7 & $8,3 \%$ \\
\hline
\end{tabular}

Nota: DPO - Dia de Pós Operatório; f* frequência de casos; \% percentual representado. Mais de um sinal ou sintoma pode ter sido reportado pelo mesmo paciente

Na tabela 4 encontram-se as associações estatísticas entre fatores de risco relacionados ao paciente e ao procedimento cirúrgico com a presença de sinais de infecção no $3^{\circ}$ DPO e $10^{\circ}$ DPO, em que se evidencia a relação significativa entre os sinais e sintomas de dor, hiperemia, edema local, febre e drenagem purulenta. 
Tabela 4. Associações estatísticas entre fatores de risco para Infecção do Sítio Cirúrgico e a presença de sinais de infecção no terceiro e décimo Dias de Pós-operatório. Picos, Piauí, Brasil, 2017.

\begin{tabular}{|c|c|c|c|c|c|c|}
\hline \multirow[t]{2}{*}{ DPO } & \multirow[t]{2}{*}{ Fatores de risco para ISC } & Dor & Hiperemia & $\begin{array}{c}\text { Edema } \\
\text { local }\end{array}$ & Febre & $\begin{array}{l}\text { Drenagem } \\
\text { purulenta }\end{array}$ \\
\hline & & $f$ & $f$ & $f$ & $\mathbf{F}$ & $f$ \\
\hline \multirow[t]{10}{*}{$3^{\circ}$} & Relacionados ao paciente & & & & & \\
\hline & Idade $>\mathbf{5 0}$ anos & 21 & 21 & & & \\
\hline & & $p=0,031$ & $p=0,000$ & & & \\
\hline & Diabetes & & & 8 & 3 & \\
\hline & & & & $p=0,009$ & $p=0,036$ & \\
\hline & Tabagismo & & 10 & & & 4 \\
\hline & & & $p=0,026$ & & & $p=0,007^{*}$ \\
\hline & $\begin{array}{l}\text { Relacionados ao } \\
\text { procedimento cirúrgico }\end{array}$ & & & & & \\
\hline & $<2$ horas & & & & & 79 \\
\hline & & & & & & $p=0,000$ \\
\hline \multirow[t]{5}{*}{$10^{\circ}$} & Relacionados ao paciente & & & & & \\
\hline & Idade $>50$ anos & & 12 & & & \\
\hline & & & $p=0,003$ & & & \\
\hline & Diabetes & 7 & 6 & 5 & & \\
\hline & & $p=0,038$ & $p=0,000$ & $p=0,025$ & & \\
\hline
\end{tabular}

Nota: DPO - Dia de Pós Operatório; * Refere-se à razão de verossimilhança, os demais valores de $p$ se referem $a 0 x^{2}$.

Independentemente do DPO, os sinais de infecção estiveram associados apenas aos fatores de risco relacionados aos pacientes. Pacientes com idade superior a 50 anos no $3^{\circ}$ DPO $(n=21 ; p=0,031)$ representaram, o fator de risco com maior associação aos sinais de infecção, sendo eles dor e hiperemia. Fato reiterado no $10^{\circ} \mathrm{DPO}$ com a ocorrência de $12(p=0,003)$ casos de hiperemia entre pacientes com mais de 50 anos.

As cirurgias com duração inferior a duas horas estiveram associadas a não ocorrência de drenagem purulenta. No décimo DPO, os pacientes com diabetes tiveram dor $(n=7 ; p=0,038)$, edema local $(n=5 ; p=0,025)$ e hiperemia. Em todos os casos o valor de $p$ foi menor que 0,05 .
Independentemente do DPO, os sinais de infecção estiveram associados apenas aos fatores de risco relacionados aos pacientes. Pacientes com idade superior a 50 anos no $3^{\circ} \mathrm{DPO}(\mathrm{n}=\mathbf{2 1}$; $\mathrm{p}=0,031$ ) representaram, o fator de risco com maior associação aos sinais de infecção, sendo eles dor e hiperemia. Fato reiterado no $10^{\circ}$ DPO com a ocorrência de $12(p=0,003)$ casos de hiperemia entre pacientes com mais de $\mathbf{5 0}$ anos.

Dessa forma, foi possível perceber que no terceiro DPO, a idade, o diagnóstico de diabetes e o hábito de fumar foram os fatores de risco que apareceram mais vezes nas associações com os sinais de infecção. Já no $10^{\circ}$ DPO, o diabetes foi o fator de risco que apareceu mais vezes nas associações com os sinais de infecção, estando mais relacionado a infecções a longo prazo. Em ambos os períodos, a hiperemia foi o sinal de infecção mais frequente.

\section{Discussão}

Um aumento progressivo do número de intervenções cirúrgicas em ortopedia e traumatologia tem ocorrido nas últimas décadas ${ }^{12}$, com isso aumenta-se a preocupação com as Infecções do Sítio Cirúrgico (ISC) cujas complicações incide em âmbito financeiro e social. Trata-se de uma 
interação complexa e multifacetada entre fatores extrínsecos e intrínsecos, conferindo especificidade individual quanto ao risco de desenvolvimento de infecção.

A caracterização sociodemográfica deste estudo revela que a maioria dos procedimentos cirúrgicos traumato-ortopédicos é realizada em homens adultos jovens na faixa etária de 18 a 59 anos. Em literatura com o mesmo delineamento metodológico nota-se a prevalência de ISC entre 19 a 92 anos, sendo 62,5\% desses pacientes entre 18 e 48 anos $^{8}$, e dentre 21 a 86 anos, com maior número de sujeitos entre 60 a 70 anos de idade ${ }^{13}$. Nas duas situações o público pertenceu majoritariamente ao sexo masculino.

Complementando esses achados, em uma pesquisa realizada em Picos, 88,7\% dos acidentes automobilísticos com trauma envolvia indivíduos do sexo masculino ${ }^{14}$. Tal incidência está relacionada a uma maior vulnerabilidade do homem a violência urbana por representarem a maior parcela de condutores de carros e motocicletas ${ }^{15}$, fundamentando os resultados encontrados neste estudo e em semelhantes.

Quanto ao período de internação pré-operatória, o risco para ISC deve considerar o tempo decorrido até a cirurgia, bem como o estabelecimento de um ambiente adequado para manter o sítio cirúrgico saudável ${ }^{16}$. Na instituição onde ocorreu a pesquisa, é comum o funcionamento da unidade comportando usuários de diferentes níveis de complexidade e faixa etária numa mesma enfermaria, devido à estrutura incompatível com a demanda do serviço, configurando um local essencialmente que potencialize os riscos para infecção.

Desse modo, o período de internação torna-se proporcional ao risco de ISC, uma vez que a ausência de Sala de Recuperação Pós Anestésica conduz os pacientes em pós-operatório imediato ao repouso nas enfermarias compartilhada com demais pacientes da instituição. Assim, a não segregação apropriada pode aumentar as chances destes a serem colonizados por flora bacteriana hospitalar, intensificando a vulnerabilidade para o surgimento de infecções nosocomiais, especialmente as infecções de sítio cirúrgico.

Nesta amostra, a estadia pré-operatória apresentou variação entre 1 e 20 dias, com média de cinco dias, sendo que $32,1 \%(n=27)$ dos pacientes passaram por período superior a 4 dias. Tais dados vão de encontro a realidade de outros dois hospitais brasileiros, em que o período de internação pré-operatória teve um tempo máximo de 30 dias $^{17}$ e média de cinco dias ${ }^{18}$. Em estudo com número amostral semelhante ao desta pesquisa, o período de internação hospitalar representou um fator de risco para a ocorrência de ISC mesmo no pós-operatório ${ }^{19}$.

Considerando que a ocorrência de ISC tem sido maior em pacientes internados de um a três dias $^{8}$, uma análise das condições envolvidas na permanência prolongada na instituição deve ser realizada a fim de identificar disfunções modificáveis. Nesse seguimento, compreende-se que a rotina como o serviço se organiza, quadro de funcionários atuantes, recursos humanos e financeiros, são fatores determinantes nesse processo. O resultado encontrado não atende as recomendações para prevenir infecções que apontam uma estadia ideal menor ou equivalente a 24 horas $^{20}$.

Como fator de risco para ISC relacionado ao paciente, as comorbidades foram relatadas em 8,3\% dos casos analisados, destacando-se o Diabetes Mellitus (DM), que tem se sobressaído em meio as cirurgias ortopédicas por prejudicar os processos inflamatórios e de cicatrização ${ }^{21}$. No manejo de fraturas de tornozelo o DM foi responsável pelo aumento de complicações, cicatrização tardia da ferida operatória e taxas de infecção variando entre $10 \%$ a $60 \%$ 22-23. 
O estresse gerado pela cirurgia e alterações no padrão alimentar às vésperas do procedimento contribuem para maior resistência à insulina e estabelecimento de quadro hiperglicêmico no perioperatório ${ }^{24}$. Visto isso, a literatura aponta que durante as primeiras 24 horas de pós-operatório o índice glicêmico não deve ultrapassar $200 \mathrm{mg} / \mathrm{dl}$ a fim de não prejudicar a capacidade de defesa do organismo ${ }^{25}$.

Nessa pesquisa, o DM se comportou como importante fator de risco para o desenvolvimento de ISC, estando relacionado com os sinais de infecção de dor, hiperemia e edema local. Vale ressaltar que, como condição crônica muitas vezes sujeita ao uso contínuo de medicamento oral, o Diabetes pode representar um fator de risco a

Nessa pesquisa, o DM se comportou como importante fator de risco para o desenvolvimento de ISC, estando relacionado com os sinais de infecção de dor, hiperemia e edema local. ser potencializado pelas interações medicamentosas passíveis de ocorrer durante a estadia hospitalar, sendo que 24 casos analisados (28,6\%) faziam uso de medicamentos habitualmente, como: corticoides, anticoagulantes e imunossupressores. Observa-se ainda, que para pacientes diabéticos insulinodependentes o risco de desenvolver infecção de sítio cirúrgico é ampliado em três vezes para cirurgias ortopédicas ${ }^{26}$.

As associações estatísticas entre fatores de risco para ISC e sinais de infecção no $3^{\circ}$ e $10^{\circ}$ DPO, mostra que os sinais de infecção estão predominantemente associados aos fatores de risco relacionados aos pacientes em comparação aos relacionados ao procedimento cirúrgico. Tal resultado aponta para a necessidade de esforços direcionados a sensibilização dos pacientes que irão passar pela intervenção cirúrgica, possibilitando minoração do risco de infecção e melhoria das chances de uma recuperação breve e sem complicações.

Com relação ao tabagismo, obteve-se $25 \%$ de casos semelhante a outros cenários em que o mesmo fator gerou valor de $p$ significativo ${ }^{12,27}$ e indicaram que a ocorrência de ISC é mais comum em pacientes fumantes se comparado aqueles que não fumam ${ }^{28}$. Os sinais de ISC relacionadas ao tabagismo aqui foram caracterizados por dor, hiperemia e drenagem purulenta. Considerando os fatores de risco inerentes ao paciente, a drenagem purulenta esteve presente apenas em pacientes tabagistas. A suspensão do tabagismo por um período mínimo de quatro semanas que antecedem a operação é favorável à redução de complicações decorrentes do uso contínuo de tabaco ${ }^{16}$.

O uso de tabaco exprime a principal causa de morte evitável no Brasil e no mundo. Cerca de metade da população fumante tenta parar o hábito, mas apenas uma parcela mínima recebe orientação e acompanhamento de um profissional de saúde adequadamente ${ }^{29}$. Com isso, pode-se inferir que este fator de risco para infecção é reflexo de um problema social de ampla magnitude, incumbindo aos profissionais e gestores de saúde a elaboração e implementação de medidas eficazes em sensibilizar e modificar o comportamento dos usuários quanto aos riscos de tal prática para a saúde.

Representando o fator de risco intrínseco ao paciente mais evidente nesta amostra, a idade superior a 50 anos também tem sido referida em diferentes realidades pesquisadas ${ }^{9,25}$, aqui esteve relacionada aos sinais e sintomas de dor e hiperemia no $3^{\circ} \mathrm{DPO}$ e hiperemia no $10^{\circ}$. A 
ausência de quadro febril em pacientes idosos pode ser consequência do sistema imunológico fisiologicamente menos ativo nessa fase, prejudicando o combate sistêmico da infecção e sua reabilitação.

Corroborando com tal ideia, estudo aponta que a fragilidade do sistema de defesa após essa faixa etária tem parcela significativa no aumento do risco de desenvolver ISC em cirurgias traumato-ortopédicas ${ }^{8}$. No entanto, a presença de comorbidades nesta faixa etária também deve ser considerada como contribuinte para a ocorrência de ISC, de modo que a idade superior a 50 anos se comporta como fator de risco ampliado pela polivalência de condições debilitantes dos sujeitos.

No mesmo princípio, em pesquisa realizada numa Clínica de Ortopedia e Traumatologia, os pacientes com mais de 60 anos obtiveram as infecções após cirurgia ortopédica em associação com DM ${ }^{12}$. À vista disso, por se tratar de um fator de risco não modificável há que se considerar recursos externos para aumentar a segurança cirúrgica na população idosa. Dessa forma, sugere-se um acompanhamento rigoroso desses pacientes compreendendo da admissão à alta hospitalar, com a categorização ordenada dos medicamentos de uso habitual e execução de plano de cuidados específico e diligente.

Embora os sinais de infecção tenham se apresentado mais relacionados aos fatores de risco pertencentes ao paciente, cirurgias com duração maior que 2 horas influenciaram o desenvolvimento da ISC. Na Suiça, a presença de ISC associada a drenos esteve condicionada a duração da cirurgia $(p=0,01)^{30}$. Cirurgia com tempo superior a 120 minutos tem sido duplamente relacionada a ocorrência de ISC, pelo tempo de exposição dos tecidos ao ambiente externo e pelo cansaço da equipe de profissionais, favorecendo falhas técnicas que interferem na defesa sistêmica do corpo ${ }^{5}$. Em outro estudo, a experiência do cirurgião é apontada como fator que pode aumentar o risco de ISC, pois cirurgiões menos experientes demandam maior período de tempo, prolongando a possibilidade de entrada microbiológica para a parte interna dos tecidos $^{17}$.

Recursos humanos e institucionais são inerentes à busca por assistência à saúde com mínimas oportunidades de danos ao paciente ${ }^{32}$ e, segundo recomendações de enfermeiros, tais recursos são necessários também para a segurança do paciente em centro cirúrgico ${ }^{33}$. Nesse sentido, a aptidão dos profissionais que trabalham no centro cirúrgico é de extrema relevância para o êxito dos procedimentos realizados. A propriedade com que os aspectos técnico-científico e ético são ali exercidos, são decisivos para um desfecho favorável ou não do paciente, dado que uma linha tênue pode ser o marco determinante entre a incisão ideal e a perfuração que ameaça a vida.

Quanto ao preparo da pele do paciente, o procedimento adequado compreende a antissepsia praticada pelo profissional, a técnica e produto utilizado. Nesta pesquisa, não houve registros de tricotomia, ou por não ter sido realizada ou por subnotificação ${ }^{21}$. Acerca da antissepsia optou-se por lodopolividona (PVPI) tópica e degermante em detrimento da solução de Clorexidina a 2\% que deve ser a primeira opção de escolha ${ }^{34}$, comprometendo a antissepsia do local de incisão e aumentando a vulnerabilidade dos pacientes para infecção de sítio cirúrgico.

Resultado semelhante foi encontrado em outro estudo, com 86,6\% da antissepsia pré-operatória realizada com PVPI alcoólica e 2,2\% com PVPI aquoso ${ }^{25}$. Fundamental importância deve ser dada ao tratamento correto para a microbiota natural proveniente dessa região visando minimizar os riscos de infecção. Entretanto, nota-se real prevalência do uso de fórmulas que não possuem o melhor índice de descolonização bacteriana e efeito residual, fato possivelmente ocasionado pela escolha das instituições de saúde segundo o critério financeiro disponível. 
Referente a antibioticoterapia profilática, sua influência é considerada pelo rompimento da barreira epitelial durante a intervenção cirúrgica, que desencadeia reações sistêmicas e provocam o processo infeccioso $0^{6}$. Na presente amostra todos receberam a medida preventiva, indo de acordo com outras pesquisas em que $97 \%{ }^{(17)}$ e $91,8 \%{ }^{33}$ dos casos analisados receberam o antibiótico. Nesse sentido, a antibioticoprofilaxia configura-se como a estratégia pertinente ao controle de ISC mais adotado pelas instituições de saúde. Entretanto, embora todos os pacientes tenham recebido o antibiótico, a média de tempo entre a administração e início da cirurgia foi de 15 a 25 minutos, contrariando a recomendação de 60 minutos antes do procedimento ${ }^{20}$, preferencialmente anterior à indução anestésica ${ }^{21}$.

Caracterizando o grau de contaminação predominante nas cirurgias analisadas, 75\% ( $n=63)$ eram limpas, estando menos propensas ao desenvolvimento de ISC, do que as demais classificações, resultado semelhante ao de outros pesquisadores ${ }^{1}$. O mesmo percentual foi alcançado para o uso de materiais ortopédicos ( $n=63 ; 75 \%)$, memorando a margem de 90 dias após o implante para ocorrência de ISC, não permitindo associar esse fator de risco com ocorrências de ISC já que o seguimento desta amostra se deu até o $10^{\circ} \mathrm{DPO}^{20}$.

Ademais, outros fatores relacionados ao paciente foram ausentes na amostra pesquisada, tais como a presença de neoplasias e imunossupressão, ou estiveram presente mas não foram significativos, como obesidade, desnutrição e alergia medicamentosa.

Alguns aspectos podem ser pontuados como limitações para esta pesquisa, sendo eles: a impossibilidade de avaliação de aspectos relacionados ao procedimento cirúrgico no momento de sua ocorrência como a paramentação cirúrgica adequada, dergermação da pele do profissional e quantidade de pessoas na sala durante o procedimento, e preparo adequado do paciente. Outros fatores são a incompletude dos dados da ficha de sala e o fato de ser um estudo unicêntrico, de modo que se outros locais estivessem envolvidos maiores contribuições poderiam ser geradas pelo cenário distinto.

\section{Conclusões}

Os sinais de infecção estiveram associados apenas aos fatores de risco relacionados aos pacientes, e os principais fatores foram a idade acima de 50 anos, diabetes e o tabagismo. Os sinais e sintomas mais prevalentes foram dor, edema e hiperemia, tanto no $3^{\circ}$ quanto no $10^{\circ}$ DPO. Ressalta-se a importância de toda a estrutura hospitalar adequada, recursos materiais e humanos para garantia da assistência cirúrgica segura, compreendendo técnica, instrumentos e práticas durante todo o tempo de estadia do paciente.

Nota-se escassez de literatura sobre os sinais de infecção apresentados pelos respectivos fatores de risco dos pacientes. Assim, espera-se que esse estudo possa contribuir para investigações futuras nessa área, avaliando o impacto destas ações após serem implementadas, como também servir de base para orientar as ações das Comissões de Infecção Hospitalar.

\section{Referências}

1. Franco LMC, Ercole FF, Mattia AD. Surgical infection in patients undergoing orthopedic surgery with implant. Rev SOBECC. 2015;20(3):163-70. https://doi.org/10.5327/Z1414-4425201500030007

2. Brasil. Ministério da Saúde. Agência Nacional de Vigilância Sanitária. Critérios Diagnósticos de Infecções Relacionadas à Assistência à Saúde. Brasília: Anvisa; 2017. 
3. Laloto TL, Gemeda DH, Abdella SH. Incidence and Predictors of Surgical Site Infection in Ethiopia: Prospective Cohort. BMC Infect Dis. 2017;17(1):119.

https://doi.org/10.5327/Z1414-4425201500030007

4. Fusco SFB, Massarico NM, Alves MVMFF, Fortaleza CMCB, Pavan ECP, Palhares VC, et al. Surgical site infection and its risk factors in colon surgeries Infección de sitio quirúrgico y sus factores de riesgo en cirugías de colon. Rev Esc Enferm USP. 2016;50(1):43-9. https://doi. org/10.1590/S0080-623420160000100006

5. Ercole FF, Franco LMC, Macieira TGR, Wenceslau LCC, Resende HIN, Chianca TCM. Risco para infecção de sítio cirúrgico em pacientes submetidos a cirurgias ortopédicas. Rev. LatinoAm. Enfermagem. 2011;19(6):1-8. http://www.scielo.br/pdf/rlae/v19n6/pt_12.pdf

6. Badia JM, Casey AL, Petrosillo N, Hudson PM, Mitchell SA, Crosby C. Impact of surgical site infection on healthcare costs and patient outcomes: a systematic review in six european countries. J Hosp Infec. 2017;96(1):1-15. https://doi.org/10.1016/j.jhin.2017.03.004

7. Mistry JB, Naqvi A, Chughtai M, Gwam C, Thomas M, Higuera CA, et al. Decreasing the incidence of surgical-site infections after total joint arthroplasty. Am J Orthop. 2018;46(6):117. https://www.ncbi.nlm.nih.gov/pubmed/29309453

8. Silva AMB, Andrade D, Wysocki AD, Nicolussi AD, Haas VJ, Miranzi MAS. Conhecimento sobre prevenção e controle de infecção relacionada à assistência à saúde: contexto hospitalar. Rev RENE. 2017;18(3):353-60. https://doi.org/10.15253/2175-6783.2017000300010

9. Reese SM, Knepper B, Young HL, Mauffrey C. Development of a surgical site infection prediction model in orthopaedic trauma: The denver health model. Injury. 2017;48(12):2699704. https://doi.org/10.1016/j.injury.2017.10.011

10. Chagas MQL, Costa AMG, Mendes PHB, Gomes Júnior SC. Analysis of surgical site infections in pediatric patients after orthopedic surgery: a case-control study. Rev Paul Pediatr. 2017;35(1):18-24. https://doi.org/10.1590/1984-0462/;2017;35;1;00011

11. Organização Mundial da Saúde. Segundo desafio global para a segurança do paciente: Cirurgias seguras salvam vidas (orientações para cirurgia segura da OMS). Rio de Janeiro: Ministério da Saúde; 2009.

12. Talic A, Dzankovic F, Papovic A, Omerhodzic E. Risk factors for the surgical field infections after the osteosynthes of tibia diaphysis. Med Arch. 2017;71(5):334-7.

https://doi.org/10.5455/medarh.2017.71.334-337

13. Bellussé GC, Ribeiro JC, Campos FR, Poveda VB, Galvão CM. Risk factors for surgical site infection in neurosurgery. Acta Paul Enferm. 2015;28(1):66-73.

https://doi.org/10.1590/1982-0194201500012

14. Soares LS, Sousa DACM, Machado ALG, Silva GRF. Profile of trauma victims from motorcycle accidents assisted in a public hospital. Rev enferm UERJ. 2015;23(1):11521. https://doi.org/10.12957/reuerj.2015.15599

15. Santos LFS, Fonseca JMA, Cavalcante BLS, Lima CM. Estudo epidemiológico do trauma ortopédico em um serviço público de emergência. Cad Saúde Colet. 2016;24(4):397-403. https://doi.org/10.1590/1414-462X201600040128

16. Perry KI, Hanssen AD. Orthopaedic infection: prevention and diagnosis. J Am Acad Orthop Surg. 2017;25(1):4-6. https://doi.org/10.5435/JAAOS-D-16-00634

17. Barbosa MH, Mendes MA, Amaral JB, Mattia ALD. Occurrence of surgical site infection in a university hospital of Minas Gerais. Rev. Min. Enferm. 2009;13(3):416-22. Available from: www.reme.org.br/exportar-pdf/33/v15n2a14.pdf

18. Pereira HO, Rezende EM, Couto BRGM. Tempo de internação pré-operatório: um fator de risco para reduzir a infecção cirúrgica em fraturas de fêmur. Rev Bras Ortop. 2015;50(6):63846. https://doi.org/10.1016/j.rbo.2015.04.011 
19. Martins T, Amante LN, Virtuoso JF, Sell BT, Wechi JS, Senna CVS. Risk factors for surgical site infections in potentially contaminated surgeries. Texto Contexto Enferm. 2018;27(3):e2790016. https://doi.org/10.1590/0104-070720180002790016

20. Brasil. Ministério da Saúde. Agência Nacional de Vigilância Sanitária. Medidas de Prevenção de Infecção Relacionada à Assistência à Saúde. Brasília: Anvisa; 2017.

21. Pereira BRR, Mendoza IYQ, Couto BRGM, Ercole FF, Gouveia VR. Hip arthroplasty: surgical site infection prevention. Rev. SOBECC. 2014;19(4):181-7.

https://doi.org/10.5327/Z1414-4425201400040002

22. Sun $\mathbf{Y}$, Wang $\mathbf{H}$, Tang $\mathbf{Y}$, Zhao $\mathbf{H}$, Qin $\mathbf{S}, \mathbf{X u} \mathbf{L}$, et al. Incidence and risk factors for surgical site infection after open reduction and internal fixation of ankle fracture: a retrospective multicenter study. Medicine (Baltimore). 2018;97(7):e9901.

https://doi.org/10.1097/MD.0000000000009901

23. Lanzetti RM, Lupariello D, Venditto T, Guzzini M, Ponzo A, De Carli A, et al. The role of diabetes mellitus and bmi in the surgical treatment of ankle fractures. Diabetes Metab Res Rev. 2018;34(2). https://doi.org/10.1002/dmrr.2954

24. Akiboye F, Rayman G. Management of hyperglycemia and diabetes in orthopedic surgery. Curr Diab Rep. 2017;17(13):1-11. https://doi.org/10.1007/s1 1892-017-0839-6

25. Poveda VB, Galvão CM, Hayashida M. Análise dos fatores de risco relacionados à incidência de infecção do sítio cirúrgico em gastrocirurgias. Rev Esc Enferm USP [Internet]. 2003 [cited 2019 Fev 28];37(1):81-9. https://doi.org/10.1590/S0080-62342003000100010

26. Santos PVF, Jesus KB, Santana KISP, Nogueira EC, Cariri LS, Brito FPG. Infecção do sítio cirúrgico em pacientes no pós-operatório de cirurgias ortopédicas eletivas. Interfaces Científicas. 2017;5(2):71-9. https://doi.org/10.17564/2316-3798.2017v5n2p71-79

27.Rodrigues ALS, Miranda AC, Dourado CJC, Almeida DPR, Brito NB, Araújo RS. Evaluation of patients as a surgical site infection in a public hospital of Belém-PA. Revista Paraense de Medicina [Internet]. 2014 [cited 2019 Mar 08];28(1):23-30. Available from:

files.bvs.br/upload/S/0101-5907/2014/v28n1/a4160.pdf

28.Oliveira EM, Paula JB. Fatores associados à infecção de sítio cirúrgico em pacientes idosos submetidos à cirurgia cardíaca com esternotomia. Saúde (Santa Maria) [Internet]. 2014 [cited 2019 Mar 08]:40(1):37-44. Available from:

https://periodicos.ufsm.br/revistasaude/article/view/7894

29.Brasil. Ministério da Saúde. Estratégias para o cuidado da pessoa com doença crônica: o cuidado da pessoa tabagista. Brasília: Ministério da Saúde; 2015.

30.Mujagic E, Zeindler J, Coslovsky M, Hoffmann H, Soysal SD, Mechera R, et al. The association of surgical drains with surgical site infections - a prospective observational study. Am J Surg. 2019;217(1):17-23. https://doi.org/10.1016/j.amjsurg.2018.06.015

31.Pereira FGF, Caetano JA, Ataíde MBC, Silva RL, Néri EDR, Carvalho GCN. Environmental variables and errors in the preparation and administration of medicines. Rev Bras Enferm. 2018;71(3):1046-54. https://doi.org/10.1590/0034-7167-2016-0041

32.Gutierres LS, Santos JLG, Peiter CC, Menegon FHA, Sebold LF, Erdmann AL. Good practices for patient safety in the operating room: nurses' recommendations. Rev Bras Enferm. 2018;71(Suppl 6):2775-82. https://doi.org/10.1590/0034-7167-2018-0449

33.Gouvêa M, Novaes CO, Iglesias AC. Assessment of antibiotic prophylaxis in surgical patients at the Gaffrée e Guinle University Hospital. Rev. Col. Bras. Cir. 2016;43(4):225-34.

https://doi.org/10.1590/0100-69912016004001

34.Oliveira AC, Gama CS. What to use in preoperative skin preparation: povidone-iodine or chlorhexidine? Rev. SOBECC. 2018;23(3):155-9.

https://doi.org/10.5327/Z1414-4425201800030007 